\title{
Who will win the CRPC drug race?
}

The race is on to find a safe and effective treatment option for delaying the progression of metastatic castrationresistant prostate cancer (CRPC). Although several contenders have surfaced in recent years, a clear winner has yet to break away from the pack. The current first-line standard of care is docetaxel. Cabazitaxel and abiraterone acetate have been approved as second-line therapies for docetaxol-resistant CRPC, but alternative therapies are urgently sought.

Three separate research teams have recently published phase II safety and efficacy data for the novel antitumor agents trabectedin, tasquinimod (TASQ), and eribulin mesylate. All three drugs stabilize CRPC progression for a short period of time in a subset of patients, but their toxicity profiles are concerning.

Michaelson et al. investigated the effects of trabectedin on 58 patients with metastatic CRPC, most of whom had received previous chemotherapy. Maximal PSA decreases of $\geq 50 \%$ were seen in approximately $11 \%$ of these patients. "Only a small proportion of patients had a PSA response, but those who did clearly benefited from treatment, with increased survival in the neighbourhood of 6 months," says Michaelson. However, grade 3-4 neutropenia, leukopenia, lymphopenia, and thrombocytopenia were all relatively common, and one study cohort closed due to safety concerns. "Trabectedin is not going to enter a phase III trial for CRPC until we can predict a higher proportion of responders- $11 \%$ is simply not enough," adds Michaelson.

Treatment with eribulin mesylate had similar results. PSA responses were seen in $8.5 \%$ of docetaxel-pretreated $(n=47)$ and $22.4 \%$ of chemotherapy-naïve $(n=58)$ patients with CRPC, and the median duration of response was 3.2 months. As with trabectedin, events such as grade 3-4 neutropenia and leukopenia were relatively common. Levels of survival in the pretreated population were similar to those found for cabazitaxel ( $\approx 15$ months) and the team concluded that the 'generally favorable toxicity profile' may be of use in patients with poorer performance status.

First-line therapy with TASQ showed modest potential. Pili and colleagues monitored disease progression in 134 chemotherapy-naïve patients treated with this angiogenesis inhibitor, in a randomized, double-blind placebocontrolled study. Although changes in PSA kinetics were slight, median progressionfree survival was significantly improved in the TASQ-treated group compared to controls (7.6 versus 3.3 months). "Its toxicity profile is acceptable as compared to cytotoxic agents and makes it suitable for rational combination strategies," reports Pili.

All three researchers highlight the need to individualize or 'tailor' treatments to patients. Suggestions for future work focus on the molecular characterization of tumors to predict drug-specific sensitivity. "The key point to this study, and to many others, is that we are in desperate need of

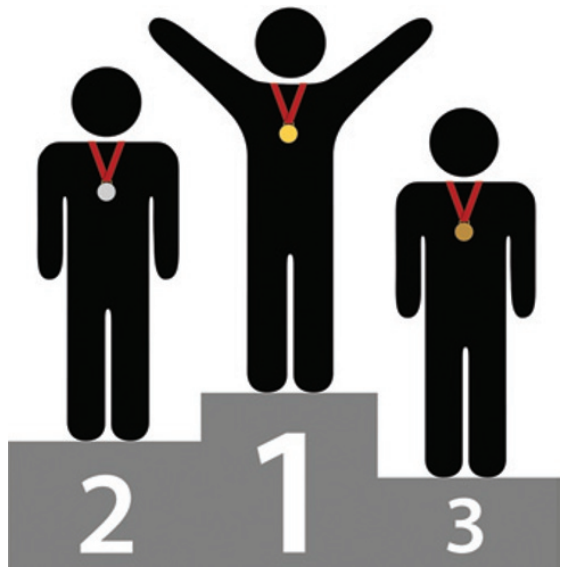

๑ Michael Brown | Dreamstime.com

biomarkers that are able to reliably predict which subsets of patients are most likely to benefit from treatment," Michaelson remarks. "If we are giving treatment with toxicity, we must be intelligent about who we give it to. We need to be able to tailor treatment to patients on an individual basis. That is the Holy Grail right now."

And so, as the race continues, perhaps the question we should be asking ourselves is: 'Are we even on the right track'?

\section{Melanie Clyne}

Original articles Michaelson, M. D. et al. Multicenter
phase II study of trabectedin in patients with metastatic
castration-resistant prostate cancer. Ann. Oncol.
doi:10.1093/annonc/mdr399 | de Bono, J. S. et al.
Phase II study of eribulin mesylate (E7389) in patients
with metastatic castration-resistant prostate cancer
stratified by prior taxane therapy. Ann. Oncol. doi:10.1093/
annonc/mdr380 | Pili, R. et al. Phase II randomized,
double-blind, placebo-controlled study of tasquinimod
in men with minimally symptomatic metastatic castrate-
resistant prostate cancer. J. Clin. Oncol. doi:10.1200/
JC0.2011.35.6295

